\title{
Pain quality descriptors and sex-related differences in patients with shoulder pain
}

This article was published in the following Dove Press journal: Journal of Pain Research

\author{
Chi-Lun Rau,' Jing-Lan Yang,' \\ Jiu-Jenq Lin, ${ }^{2,3}$ Pei-Chi Wu, ${ }^{4}$ \\ Chieh-Yi Hou, ${ }^{5}$ Chen-Yi \\ Song, ${ }^{6}$ Ching-Lin Hsieh ${ }^{2,7,8}$
}

'Department of Physical Medicine and Rehabilitation, Shuang Ho Hospital, Taipei Medical University, Taiwan; ${ }^{2}$ Department of Physical Medicine and Rehabilitation, National Taiwan University Hospital, Taiwan; ${ }^{3}$ School and Graduate Institute of Physical Therapy, College of Medicine, National Taiwan University, Taiwan; ${ }^{4}$ Department of Physical Medicine and Rehabilitation, E-Da Hospital, Taiwan; ${ }^{5}$ Department of Physical Medicine and Rehabilitation, E-Da Cancer Hospital, Taiwan; 'Department of Long-Term Care, National Taipei University of Nursing and Health Sciences, Taiwan; ${ }^{7}$ School of Occupational Therapy, College of Medicine, National Taiwan University, Taiwan; ${ }^{8}$ Department of Occupational Therapy, College of Medical and Health Science, Asia University, Taiwan
Correspondence: Chen-Yi Song Department of Long-Term Care, National Taipei University of Nursing and Health Sciences, No. 83-I, Neijiang Street, Wanhua District, Taipei City 108, Taiwan

Tel +886 22822 7lol (Extension 6136)

Fax +886 22389 I464

Email cysong@ntunhs.edu.tw
Background: Pain quality assessment is applicable to pain evaluation and treatment. However, shoulder pain quality descriptors mostly remain unknown. Furthermore, sex-related differences considerably affect clinical pain experience. The aim of this study was to investigate pain quality descriptors and to compare sex-related differences in using pain descriptors among patients with shoulder pain.

Materials and methods: A sample of 120 patients (41 males and 79 females) with shoulder pain was recruited from Department of Physical Medicine and Rehabilitation. Shoulder pain quality descriptors were investigated using a 36-item pain quality list. Sex-related differences in the number and frequency of pain quality descriptors were compared using independent $t$-test and $X^{2}$ test, respectively.

Results: Fifteen commonly used shoulder pain quality descriptors were identified. Among them, "sore" was the most frequently used, followed by "pulled". Deep pain sensations (eg, sore, pulled, torsion, and taut) were relatively more predominant than superficial pain sensations (eg, pricking and lacerating). In terms of sex-related differences, female patients used more pain quality descriptors than the male patients $(5.5 \mathrm{vs} 3.7, P<0.001)$. The frequency of paroxysmal, dullness, and constriction-related pain quality descriptors, such as "shooting", "faint", "clicking", and "squeezing", were higher in females than in males (all $P<0.05$ ).

Conclusion: The results provide commonly used shoulder pain quality descriptors that are useful for assessing shoulder pain and for developing a new shoulder pain assessment tool. Because the shoulder pain quality profiles differed between male and female participants, clinicians and researchers should consider sex-related differences in assessing and treating shoulder pain.

Keywords: pain assessment, measurement, musculoskeletal pain, culture

\section{Introduction}

Pain is an individualized, subjective, and multidimensional experience that relies considerably on communication. ${ }^{1}$ Studies on words that patients use to describe chronic pain have indicated that pain quality descriptors are the most frequently used. ${ }^{2-4}$ Therefore, pain quality descriptors are crucial in describing pain.

Implications of pain quality descriptors have received increasing attention. These descriptors have helped clinicians discriminate chronic pain conditions, ${ }^{4}$ pain mechanisms, ${ }^{5}$ and pain sensations ${ }^{6}$ and identify responses to treatments. ${ }^{7,8}$ These results suggest that pain quality assessment is applicable to pain evaluation and treatment.

Pain quality descriptors of lower back pain ${ }^{4,5,8,9}$ and arthritis-related pain ${ }^{5,8,10,11}$ have been studied. Although shoulder pain is a common musculoskeletal pain condition, ${ }^{12}$ shoulder pain quality descriptors mostly remain unknown. Because pain has 
multiple and distinct qualities, ${ }^{4}$ a variation in pain perception can be reasonably assumed among patients with different musculoskeletal diseases. Therefore, back pain and arthritic pain quality descriptors reported in previous studies cannot be generalized to shoulder pain. Furthermore, although musculoskeletal pain is traditionally considered nociceptive, several studies have suggested shared mechanisms of neuropathic pain in people with osteoarthritis and shoulder pain. ${ }^{13,14}$ Because pain quality descriptors have the potential to facilitate discrimination of pain mechanisms, ${ }^{5}$ exclusively assessing shoulder pain quality facilitates understanding nociceptive contributions and neuropathic pain components in overall shoulder pain. A mechanism-based classification of shoulder pain benefits shoulder pain management.

The language used to describe pain may vary among men and women because they may have learned different words from earlier pain experiences for describing pain, ${ }^{15}$ and studies have shown that sex-related differences considerably affect clinical pain experience. ${ }^{16,17}$ However, few studies have examined sex-related differences in pain language, particularly in the sensory quality of pain. ${ }^{1,18}$ A study in Australia showed that female university students used more words and more McGill Pain Questionnaire (MPQ) descriptors and focused more on pain quality than male students did to describe their pain experiences. ${ }^{1}$ By contrast, Robinson et al ${ }^{18}$ found no sex-related differences in sensory subscale scores of the MPQ among patients with back and facial pain, indicating similar pain quality among men and women. Hence, no conclusive sex-related difference regarding pain quality descriptors was observed.

Investigating sex-related differences in pain quality descriptors has clinical and research implications. Providing healthcare professionals with the knowledge of sex-specific pain terminologies can facilitate clinical communication with patients for pain-related assessment and treatment planning. Moreover, if sex-related differences exist, researchers should consider the differences during development of a pain quality assessment tool and the rating principles for clinical and research use.

The purpose of this study was to investigate shoulder pain quality descriptors and compare sex-related differences. We hypothesize that sex-related differences exist in the number and frequency of shoulder pain quality descriptors.

\section{Material and methods Overview of study design}

The study used a cross-sectional design in which 1) shoulder pain quality descriptors were investigated and 2) sex-related differences in the number and frequency of shoulder pain quality descriptors were compared.

\section{Participants}

The study included 120 patients who were undergoing physical therapy for shoulder pain in the Department of Physical Medicine and Rehabilitation at National Taiwan University (NTU) Hospital. Eligible patients were required to 1) have a primary shoulder diagnosis (eg, adhesive capsulitis, rotator cuff tear, or tendinitis), 2) be 18 years or older, and 3) use Mandarin Chinese as their primary language. Exclusion criteria were 1) presence of a concomitant illness likely to confound pain assessment (eg, a wound or an infection), 2) presence of a serious or unstable medical or psychological condition (eg, stroke, cancer, depression, and schizophrenia) that could compromise study participation, and 3) surgery within the past 30 days. The participants provided signed informed consent form prior to participation. The study protocol was approved by the Ethics Committee of the NTU Hospital.

\section{Data collection}

To obtain a comprehensive list of shoulder pain quality descriptors for prompting the participants, 116 rehabilitation staff members (therapists, physiatrists, and nurses) having comprehensive pain vocabularies acquired through clinical practice, personal pain experiences, and medical education were surveyed regarding Chinese pain quality descriptors. Staff members with clinical experiences exceeding 2 years and who were regularly involved in the care of patients with pain were requested to provide descriptors to describe various pain syndromes (Table 1). Based on their responses, 36 pain quality descriptors were obtained (Table 2), and a 36-item pain quality list was generated.

The participants were offered the 36 -item pain quality list to select pain quality descriptors that corresponded to their shoulder pain. The participants were allowed to describe shoulder pain sensation characteristics that they experienced but were not in the list. They were incentivized with US\$3 gift cards for completing the questionnaire.

In addition to pain quality, the worst pain intensity experienced in the preceding week was assessed using an 11-point rating scale from 0 (no pain) to 10 (extreme pain). ${ }^{19}$ The pain duration and symptomatic side (shoulder) were also recorded.

\section{Data analysis}

Statistical analyses were performed using SPSS 20.0 for Windows. Descriptive statistics were used to determine 
Table I List of 28 pain syndromes

\begin{tabular}{lc}
\hline Pain syndrome & Pain syndrome \\
\hline Head and neck pain & Extremity pain \\
Headache & Upper extremity joint pain \\
Toothache & Lower extremity joint pain \\
Neck pain & Musculoskeletal pain \\
Sore throat & Palindromic rheumatism \\
Shoulder pain & Gout \\
Chest pain & Muscular spasm (cramp) \\
Chest wall pain & Muscle soreness \\
Angina & Ligament sprain \\
Abdominal pain & Fracture \\
Stomach ache & Neurological pain \\
Abdominal pain & Sciatica \\
Pelvic pain & Trigeminal neuralgia \\
Menstrual pain & Intercostal neuralgia \\
Labor pain & Postherpetic neuralgia \\
Painful defecation & Other pain \\
Dysuria & Surgical wound pain \\
Back pain & \\
Backache & \\
\hline
\end{tabular}

the participants' demographics and clinical characteristics. The number and frequency of pain quality descriptors used by the participants were also calculated separately by sex. Descriptors used by more than $10 \%$ of the participants were considered common pain descriptors. ${ }^{3}$

An independent $t$-test was used to compare the continuous variables (ie, age, pain intensity, duration, and number of pain quality descriptors), and the chi-squared $\left(X^{2}\right)$ or Fisher's exact $t$-test was used to compare the categorical variables (ie, education level, diagnosis, symptomatic side, painful stage, and frequency of pain quality descriptors) between the male and female patients. Differences were considered significant at $P$ $<0.05$. Additionally, two effect size (ES) measures (Cohen's $\mathrm{d}$ and Cramér's V) were calculated to quantify the magnitude of the differences between the male and female participants. Cohen's d values of $0.20,0.50$, and 0.80 indicated small, moderate, and large ES, respectively, and Cramér's V values of $0.10,0.30$, and 0.50 were considered small, moderate, and large ES, respectively. ${ }^{20}$

\section{Results}

\section{Description of the study sample}

Among the 120 participants, 41 were males and 79 were females. The participants were similar in age, educational level, and diagnosis (Table 3 ). In addition, we found no sexrelated differences regarding the side of the shoulder pain, pain intensity, or pain duration (Table 3). Most participants were diagnosed with adhesive capsulitis (frozen shoulder), tenosynovitis, or tendinitis and experienced chronic pain with moderate pain intensity (Table 3 ).

\section{Shoulder pain quality descriptors}

Table 2 lists the number and frequency of the shoulder pain quality descriptors in the order of the total frequency among all participants. A total of 31 shoulder pain quality descriptors were identified. Participants provided no supplementary descriptors. Fifteen quality descriptors - sore, pulled, torsion, taut, faint, shooting, pricking, pressing, clicking, lacerating, pinch, numb, tension, dull, and squeezing - were used by more than $10 \%$ of the participants. The participants on average used $4.9(\mathrm{SD}=2.9)$ pain quality descriptors to describe shoulder pain.

\section{Sex-related differences in the number and frequency of shoulder pain quality descriptors}

The female participants provided more pain quality descriptors than male participants did. The average number of descriptors provided by female and male participants was 5.5 (SD 3.0) and 3.7 (SD 2.0), respectively $(P<0.001$, Cohen's d $=0.67$ ). Fifteen relatively common pain quality descriptors were used by the female participants, whereas 11 were used by the male participants (Table 2).

"Sore" was the most common pain quality descriptor used by participants of both sexes, followed by "pulled". The frequency of using other pain quality descriptors varied among men and women (Table 2). Four pain quality descriptors, "faint", "shooting", "clicking", and "squeezing", were used frequently by the female participants (all $P<0.05$, Cramér's $\mathrm{V}=0.19-0.26$ ) along with "tension" and "numb" (both $P=$ 0.05 , Cramér's V $=0.18$ ).

\section{Discussion}

The current study discovered 15 common shoulder pain quality descriptors and identified different patterns or profiles of shoulder pain quality between male and female participants. The findings contribute to the growing research on various experiences of pain quality from a patient's perspective. Because sex-related differences were observed in pain quality but not in pain intensity, our findings suggest that the scores based on global pain intensity may fail to reflect the diversity of pain manifestations and the complexity of underlying biological mechanisms in men and women.

Shoulder pain was found to have a diverse pain quality (Table 2), comprising deep and superficial pain characteristics. ${ }^{9,11}$ Deep pain entails traction (eg, pulled and torsion), 
Table 2 Frequency of pain quality descriptors among male $(N=4 I)$ and female $(N=79)$ patients with shoulder pain

\begin{tabular}{|c|c|c|c|c|}
\hline $\begin{array}{l}\text { Pain quality } \\
\text { descriptor }\end{array}$ & $\begin{array}{l}\text { Total } \\
\text { n (\%) }\end{array}$ & $\begin{array}{l}\text { Male } \\
\text { n (\%) }\end{array}$ & $\begin{array}{l}\text { Female } \\
\text { n (\%) }\end{array}$ & $P$-value \\
\hline Sore & $78(65.0)$ & $24(58.5)$ & $54(68.4)$ & 0.285 \\
\hline Pulled & $61(50.8)$ & $20(48.8)$ & $4 \mid$ (5I.9) & 0.746 \\
\hline Torsion & $49(40.8)$ & 15 (36.6) & $34(43.0)$ & 0.495 \\
\hline Taut & $45(37.5)$ & II (26.8) & $34(43.0)$ & 0.082 \\
\hline Faint* & 44 (36.7) & $8(19.5)$ & $36(45.6)$ & 0.005 \\
\hline Shooting* & $38(31.7)$ & $8(19.5)$ & $30(38.0)$ & 0.039 \\
\hline Pricking & $36(30.0)$ & $9(22.0)$ & $27(34.2)$ & 0.166 \\
\hline Pressing & $35(29.2)$ & II (26.8) & $24(30.4)$ & 0.685 \\
\hline Clicking* & $35(29.2)$ & 7 (I7.I) & $28(35.4)$ & 0.036 \\
\hline Lacerating & $23(19.2)$ & $9(22.0)$ & 14 (I7.7) & 0.577 \\
\hline Pinch & $18(15.0)$ & $5(12.2)$ & $13(16.5)$ & 0.535 \\
\hline Numb & $16(13.3)$ & $2(4.9)$ & 14 (I7.7) & 0.050 \\
\hline Tension & $16(13.3)$ & $2(4.9)$ & 14 (I7.7) & 0.050 \\
\hline Dull & I4 (I I.7) & $2(4.9)$ & $12(15.2)$ & 0.135 \\
\hline Squeezing* & 14 (II.7) & I (2.4) & $13(16.5)$ & 0.033 \\
\hline Sharp & $10(8.3)$ & $4(9.8)$ & $6(7.6)$ & 0.734 \\
\hline Drilling & $8(6.7)$ & $2(4.9)$ & $6(7.6)$ & 0.714 \\
\hline Spasm & $7(5.8)$ & $2(4.9)$ & $5(6.3)$ & 1.000 \\
\hline Percussion & $7(5.8)$ & $2(4.9)$ & $5(6.3)$ & 1.000 \\
\hline Throbbing & $5(4.2)$ & I (2.4) & $4(5.1)$ & 0.660 \\
\hline Hot & $5(4.2)$ & $0(0)$ & $5(6.3)$ & 0.164 \\
\hline Burning & $5(4.2)$ & $0(0)$ & $5(6.3)$ & 0.164 \\
\hline Cramping & $4(3.3)$ & $2(4.9)$ & $2(2.5)$ & 0.605 \\
\hline Cutting & $3(2.5)$ & $0(0)$ & $3(3.8)$ & 0.550 \\
\hline Explosive & $2(1.7)$ & $0(0)$ & $2(2.5)$ & 0.546 \\
\hline Cold & $2(1.7)$ & $0(0)$ & $2(2.5)$ & 0.546 \\
\hline Heavy & $2(1.7)$ & I (2.4) & $\mathrm{I}(\mathrm{I} .3)$ & 1.000 \\
\hline Itchy & $2(1.7)$ & I (2.4) & I (I.3) & 1.000 \\
\hline Dizziness & I (0.8) & $0(0)$ & $\mathrm{I}(1.3)$ & 1.000 \\
\hline Radiating & I (0.8) & $0(0)$ & $\mathrm{I}(\mathrm{I} .3)$ & 1.000 \\
\hline Scratchy & I (0.8) & I (2.4) & $0(0)$ & 0.342 \\
\hline Dry & $0(0)$ & $0(0)$ & $0(0)$ & - \\
\hline Electrical & $0(0)$ & $0(0)$ & $0(0)$ & - \\
\hline Distension & $0(0)$ & $0(0)$ & $0(0)$ & - \\
\hline Corrosive & $0(0)$ & $0(0)$ & $0(0)$ & - \\
\hline Stinging & $0(0)$ & $0(0)$ & $0(0)$ & - \\
\hline
\end{tabular}

Note: "The frequency of this pain descriptor was higher in females than in males $(P<0.05)$.

dullness (eg, sore, dull, faint, or slight), and constriction (eg, taut and squeezing) sensations, whereas superficial pain comprises puncturing or abrasive (eg, pricking), incisive (eg, lacerating), ${ }^{9,21}$ and numbness sensations. ${ }^{11}$ The presence of deep and superficial pain sensations (eg, dull, pressing, and pricking) suggests that shoulder pain is conducted through A and $\mathrm{C}$ nerve fibers, the major pain-conducting nerve fibers. ${ }^{6}$

In addition to deep and superficial pain sensations, we found that paroxysmal pain sensations (eg, shooting) ${ }^{11}$ were frequently ( $>30 \%$ ) noted in patients with shoulder pain; by contrast, "hot" and "cold" were rarely used (<5\%). Similar findings were observed in a recent study on pain quality response profiles of treatment for shoulder impingement syndrome, which showed high intensities of deep (eg, dull and achy) or paroxysmal (eg, sharp and shooting) pain quality and relatively minor "hot" or "cold" pain at the baseline. The descriptor "cold" appears to be seldom used to describe musculoskeletal pain. Studies on lower back and knee pain have also found that the descriptor "cold" was rarely used., 4,5, Combining the 15 common shoulder pain quality descriptors facilitates the study of shoulder pain of different etiologies.

Various pain qualities are associated with different underlying pain mechanisms. ${ }^{8}$ Deep pains (eg, sore, pulled, and torsion), which are relatively predominant in nociceptive pain, ${ }^{5}$ are commonly used as shoulder pain descriptors. Although shoulder pain is traditionally considered nocicep- 
Table 3 Demographic and clinical characteristics of patients with shoulder pain

\begin{tabular}{|c|c|c|c|}
\hline Characteristic & Male $(n=4 I)$ & Female $(n=79)$ & $P$-value \\
\hline Age (y) & $52.6 \pm 15.0$ & $55.1 \pm 8.7$ & 0.315 \\
\hline Height (cm) & $169.9 \pm 6.4$ & $157.0 \pm 5.4$ & - \\
\hline Weight (kg) & $69.8 \pm 10.7$ & $56.2 \pm 8.9$ & - \\
\hline Education level $^{a}$ & & & 0.191 \\
\hline Primary school & 4 & 5 & \\
\hline Junior high school & 2 & 8 & \\
\hline Senior high school & 2 & 9 & \\
\hline Professional school & 3 & 10 & \\
\hline Junior college & 3 & 12 & \\
\hline College & 13 & 22 & \\
\hline Graduate school & 14 & 12 & \\
\hline Diagnosis & & & 0.725 \\
\hline Adhesive capsulitis/frozen shoulder & 15 & 32 & \\
\hline Tenosynovitis/tendinitis & 10 & 20 & \\
\hline Rotator cuff tear (syndrome) & 5 & 5 & \\
\hline Sprain & 6 & 6 & \\
\hline Fracture & 0 & 1 & \\
\hline Bursitis & 2 & 5 & \\
\hline Arthritis & 3 & 10 & \\
\hline Symptom side & & & 0.106 \\
\hline Right & 24 & 37 & \\
\hline Left & 13 & 39 & \\
\hline Bilateral & 4 & 3 & \\
\hline Pain duration $(\mathrm{mo})^{\mathrm{b}}$ & $8.9 \pm 11.8$ & $7.8 \pm 5.4$ & 0.453 \\
\hline Stage & & & 0.622 \\
\hline Acute/subacute $(0-3 \mathrm{mo})$ & 7 & 9 & \\
\hline Short-term chronic (3-12 mo) & 28 & 60 & \\
\hline Long-term chronic (>12 mo) & 6 & 10 & \\
\hline Pain intensity ( $0-10$ numeric rating scale) & $4.5 \pm 2.0$ & $5.4 \pm 2.6$ & 0.070 \\
\hline
\end{tabular}

Note: ${ }^{\mathrm{a}, \mathrm{b}}$ One female participant with missing data.

tive, $30 \%$ and $13 \%$ of our patients described their shoulder pain as "prick" and "numb", respectively. Combined with other neuropathic pain-like descriptors (eg, burning and itchy), ${ }^{22,23}$ such pain qualities suggest a shared mechanism with neuropathic pain. A recent systemic review concluded that hypersensitivity of the central nervous system contributed to shoulder pain in a subgroup within the shoulder pain population. ${ }^{14}$ Thus, with increased awareness of central sensitization in shoulder pain, implementation of accessible and cost-effective pain quality assessment may be warranted.

Consistent with our hypothesis, sex-related differences were evident in the number and frequency of shoulder pain descriptors, whereas the demographic and clinical characteristics (eg, education level, diagnosis, pain duration, and intensity) were comparable between male and female patients. The findings were similar to those of the study conducted in Australia. ${ }^{1}$ However, Robinson et $\mathrm{al}^{18}$ found no sex-related differences in pain quality among patients with back and facial pain. The discrepancy between Robinson et al's study and the current study may be attributable to the differences in patient sampling and testing methods (questionnaire usage). Nevertheless, our findings support sex-related differences in clinical pain experiences, specifically regarding shoulder pain quality, in addition to prevalence of pain in the shoulder.

The current study found that more shoulder pain quality descriptors were used by the female patients than by the male patients (5.5 vs 3.7 ) with moderate ES. We also discovered that several deep pain (eg, dullness and constriction-related sensations), paroxysmal pain (eg, shooting), and numb pain sensations were frequently reported by the female patients, although the differences appeared minor (small ES). Sexstereotyped expectations among men and women may have a strong influence on pain response. ${ }^{24}$ Theoretically, men and women are predisposed to respond differently and have different expectations relative to pain perception. Men are expected to be less sensitive, more enduring, and reluctant to report pain than women. ${ }^{24}$ Men strive to maintain the stereotype of toughness and stoicism and were reticent to admit discomfort 
because of their masculinity. In addition, Confucianism, a system of ethics and philosophy inherent in the Chinese culture, may accentuate sex differences by promoting stoicism in males and allowing greater emotional expression and reports of physical distress in females. ${ }^{25}$ Sex role expectations may explain the differences in using shoulder pain descriptors. Although why female and male patients use different shoulder pain descriptors remain unclear, the preliminary findings suggest that future research should consider sex-related differences in studying shoulder pain, particularly pain quality.

A partially open Chinese pain quality questionnaire was used to complete a comprehensive survey of shoulder pain quality descriptors, wherein patients were also allowed to provide voluntary descriptors. Several potential pain quality measures may be selected, including the MPQ and the pain quality assessment scale (PQAS). ${ }^{26,27}$ However, direct translations of measures that are developed using samples of patients from one country or culture are not necessarily content valid for use in other countries or cultures. ${ }^{28}$ Although the MPQ has 42 sensory descriptors, most were rarely used by patients with pain. ${ }^{3,4}$ Although the PQAS have demonstrated content validity as measures of pain quality in the western chronic pain populations, ${ }^{3,4}$ it contains the most common sensory descriptors, except "sore". Because "sore" (suantong in Chinese) is a Chinese culture-specific descriptor of bone, muscle, and joint pain, ${ }^{29}$ the use of the PQAS was not considered in the current investigation. Future studies can reproduce the current study design in additional samples of patients with pain, and use the identified descriptors to further develop a specific (eg, shoulder pain) or generic pain quality assessment tool. Improving pain assessment by measuring pain quality in addition to pain intensity can completely capture a patient's pain experience and response to pain treatment. ${ }^{30}$

This study has certain limitations. First, the investigation was conducted in clinical settings. Therefore, participants were patients who actively sought medical care for their shoulder pain. Physical therapy may somewhat affect their pain assessments. Second, the sample was heterogeneous consisting of distinctly different musculoskeletal conditions. Third, convenience sampling was adopted, through which more female patients were enrolled. Although this may challenge the obtained sex-related differences, the study nevertheless provides a favorable clinical picture that musculoskeletal pain, including shoulder pain, is generally highly prevalent in women. ${ }^{17}$ Finally, pain descriptors might be country-, language-, ethnicity-, or culture-specific. ${ }^{29,31}$ Therefore, the findings may not be generalized to other populations. Future studies should be conducted to explore correlations between pain quality, pain intensity, and motor function (eg, functional limitations and/or disability).

\section{Conclusion}

This study found 15 common descriptors for shoulder pain quality assessment. Because shoulder pain quality profiles differed between men and women in this study, clinicians and researchers should consider sex-related differences during shoulder pain management. Valid pain quality assessment tools are warranted in additional studies to further confirm the sex-related differences.

\section{Acknowledgments}

This study was funded by the Ministry of Science and Technology (MOST 103-2314-B-002-012 -MY3) and the E-Da Hospital (EDAHT106023), Taiwan. We thank the study participants, and we also thank Guan-Yu Chen for assistance in data management.

\section{Author contributions}

CLR and CLH - conception, study design, and manuscript writing. JLY and JJL - data analysis and manuscript writing. PCW and $\mathrm{CYH} \mathrm{-} \mathrm{data} \mathrm{collection} \mathrm{and} \mathrm{manuscript} \mathrm{writing.}$ CYS - conception, study design, data analysis, and manuscript writing. All authors contributed toward revising the paper, gave final approval of the version to be published, and agree to be accountable for all aspects of the work.

\section{Disclosure}

The authors report no conflicts of interest in this work.

\section{References}

1. Strong J, Mathews T, Sussex R, New F, Hoey S, Mitchell G. Pain language and gender differences when describing a past pain event. Pain. 2009;145(1-2):86-95.

2. Dudgeon BJ, Ehde DM, Cardenas DD, Engel JM, Hoffman AJ, Jensen MP. Describing pain with physical disability: narrative interviews and the McGill Pain Questionnaire. Arch Phys Med Rehabil. 2005;86(1):109-115.

3. Lin CP, Kupper AE, Gammaitoni AR, Galer BS, Jensen MP. Frequency of chronic pain descriptors: implications for assessment of pain quality. Eur J Pain. 2011;15(6):628-633.

4. Jensen MP, Johnson LE, Gertz KJ, Galer BS, Gammaitoni AR. The words patients use to describe chronic pain: implications for measuring pain quality. Pain. 2013;154(12):2722-2728.

5. Dworkin RH, Jensen MP, Gammaitoni AR, Olaleye DO, Galer BS. Symptom profiles differ in patients with neuropathic versus nonneuropathic pain. J Pain. 2007;8(2):118-126.

6. Beissner F, Brandau A, Henke C, et al. Quick discrimination of A(delta) and $\mathrm{C}$ fiber mediated pain based on three verbal descriptors. PLoS One. 2010;5(9):e12944.

7. Jensen MP, Trudeau JJ, Radnovich R, Galer BS, Gammaitoni AR. The pain quality response profile of a corticosteroid injections and heated lidocaine/tetracaine patch in the treatment of shoulder impingement syndrome. Clin J Pain. 2015;31(4):342-348. 
8. Jensen MP, Dworkin RH, Gammaitoni AR, Olaleye DO, Oleka N, Galer BS. Assessment of pain quality in chronic neuropathic and nociceptive pain clinical trials with the Neuropathic Pain Scale. J Pain. 2005;6(2): 98-106.

9. Fernandez E, Vargas R, Mahometa M, Ramamurthy S, Boyle GJ. Descriptors of pain sensation: a dual hierarchical model of latent structure. J Pain. 2012;13(6): 532-536.

10. Lalloo C, Stinson JN, Hochman JR, Adachi JD, Henry JL. Adapting the Iconic Pain Assessment Tool Version 2 (IPAT2) for adults and adolescents with arthritis pain through usability testing and refinement of pain quality icons. Clin J Pain. 2013;29(3):253-264.

11. Victor TW, Jensen MP, Gammaitoni AR, Gould EM, White RE, Galer BS. The dimensions of pain quality: factor analysis of the Pain Quality Assessment Scale. Clin J Pain. 2008;24(6):550-555.

12. Luime JJ, Koes BW, Hendriksen IJ, et al. Prevalence and incidence of shoulder pain in the general population; a systematic review. Scand J Rheumatol. 2004;33(2): 73-81.

13. Moreton BJ, Tew V, das Nair R, Wheeler M, Walsh DA, Lincoln NB Pain phenotype in people with knee osteoarthritis: classification and measurement properties of painDETECT and self-report Leeds assessment of neuropathic symptoms and signs scale in a cross-sectional study. Arthritis Care Res (Hoboken). 2015;67(4): 519-528.

14. Sanchis MN, Lluch E, Nijs J, Struyf F, Kangasperko M. The role of central sensitization in shoulder pain: a systematic literature review. Semin Arthritis Rheum. 2015; 44(6):710-716.

15. Merskey H, Bogduk N. Classification of Chronic Pain. 2nd ed. Seattle: IASP; 1994.

16. Fillingim RB, King CD, Ribeiro-Dasilva MC, Rahim-Williams B, Riley JL 3rd. Sex, gender, and pain: a review of recent clinical and experimental findings. J Pain. 2009;10(5):447-485.

17. Unruh AM. Gender variations in clinical pain experience. Pain. 1996;65(2-3):123-167.

18. Robinson ME, Wise EA, Riley III JL, Atchison JW. Sex differences in clinical pain: a multisample study. J Clin Psychol Med Settings. 1998;5(4):413-424.
19. Ger LP, Ho ST, Sun WZ, Wang MS, Cleeland CS. Validation of the brief pain inventory in a Taiwanese population. J Pain Symptom Manage. 1999;18(5):316-322.

20. Cohen J. Statistical Power Analysis for the Behavioral Sciences. Hillsdale: Lawrence Erlbaum; 1998.

21. Fernandez E, Krusz JC, Hall S. Parsimonious collection of pain descriptors: classification and calibration by pain patients. J Pain. 2011;12(4):444-450.

22. Bennett M. The LANSS Pain Scale: the Leeds assessment of neuropathic symptoms and signs. Pain. 2001;92(1-2):147-157.

23. Bouhassira D, Attal N, Alchaar H, et al. Comparison of pain syndromes associated with nervous or somatic lesions and development of a new neuropathic pain diagnostic questionnaire (DN4). Pain. 2005;114(1-2):29-36

24. Robinson ME, Riley JL 3rd, Myers CD, et al. Gender role expectations of pain: relationship to sex differences in pain. J Pain. 2001;2(5):251-257.

25. Tsai YF. Gender differences in pain and depressive tendency among Chinese elders with knee osteoarthritis. Pain. 2007;130(1-2):188-194.

26. Melzack R. The McGill Pain Questionnaire: major properties and scoring methods. Pain. 1975;1(3):277-299.

27. Jensen MP, Gammaitoni AR, Olaleye DO, Oleka N, Nalamachu SR, Galer BS. The pain quality assessment scale: assessment of pain quality in carpal tunnel syndrome. J Pain. 2006;7(11): 823-832.

28. Sharma S, Pathak A, Jensen MP. Words that describe chronic musculoskeletal pain: implications for assessing pain quality across cultures J Pain Res. 2016;9:1057-1066.

29. Moore RA, Dworkin SF. Ethnographic methodologic assessment of pain perceptions by verbal description. Pain. 1988;34(2):195-204.

30. Dworkin RH, Turk DC, Farrar JT, et al; IMMPACT. Core outcome measures for chronic pain clinical trials: IMMPACT recommendations. Pain. 2005;113(1-2):9-19.

31. Moore R, Brødsgaard I, Miller ML, Mao TK, Dworkin SF. Consensus analysis: reliability, validity, and informant accuracy in use of American and Mandarin Chinese pain descriptors. Ann Behav Med. 1997;19(3):295-300.

\section{Journal of Pain Research}

\section{Publish your work in this journal}

The Journal of Pain Research is an international, peer reviewed, open access, online journal that welcomes laboratory and clinical findings in the fields of pain research and the prevention and management of pain. Original research, reviews, symposium reports, hypothesis formation and commentaries are all considered for publication

\section{Dovepress}

The manuscript management system is completely online and includes a very quick and fair peer-review system, which is all easy to use. Visit http://www.dovepress.com/testimonials.php to read real quotes from published authors. 